\title{
On upper bounds for infinite Prandtl number convection with or without rotation
}

\author{
Charles R. Doering \\ Department of Mathematics, University of Michigan, Ann Arbor, Michigan 48109-1109 \\ Peter Constantin ${ }^{\text {a) }}$ \\ Department of Mathematics, University of Chicago, Chicago, Illinois 60637
}

(Received 7 April 2000; accepted for publication 2 November 2000)

\begin{abstract}
Bounds for the bulk heat transport in Rayleigh-Benard convection for an infinite Prandtl number fluid are derived from the primitive equations. The enhancement of heat transport beyond the minimal conduction value (the Nusselt number $\mathrm{Nu}$ ) is bounded in terms of the nondimensional temperature difference across the layer (the Rayleigh number $\mathrm{Ra}$ ) according to $\mathrm{Nu} \leqslant c \mathrm{Ra}^{2 / 5}$, where $c<1$ is an absolute constant. This rigorous upper limit is uniform in the rotation rate when a Coriolis force, corresponding to the rotating convection problem, is included. (c) 2001 American Institute of Physics. [DOI: 10.1063/1.1336157]
\end{abstract}

\section{INTRODUCTION}

Rayleigh-Benard convection, where a fluid layer heated from below produces an instability leading to convective fluid motions, has played a central role in both the experimental and theoretical development of the modern sciences of nonlinear dynamics and physical pattern formation. Driven far beyond the instability, thermal convection becomes turbulent. Heat transport by convective turbulence is an important component of a wide variety of problems in applied physics ranging from stellar structure in astrophysics, ${ }^{1}$ to mantle convection and plate tectonics in geophysics, ${ }^{2}$ to transport in physical oceanography and atmospheric science. ${ }^{3}$ One of the fundamental quantities of interest in these systems is the total heat transport across the layer, usually expressed in terms of the nondimensional Nusselt number $\mathrm{Nu}$, the enhancement of heat flux beyond the minimal conductive value. This flux is a function of the buoyancy force across the layer, usually measured in units set by the layer geometry and material dissipation parameters as the Rayleigh number Ra. There are at least two other parameters in these systems: The Prandtl number Pr, a material parameter, is the ratio of diffusivities of momentum and temperature. And the aspect ratio $\mathcal{A}$, the ratio of the cross-sectional length scale(s) to the layer depth, is a geometric parameter characterizing the convection domain.

A major goal of both theory and experiment is to elucidate the $\mathrm{Nu}-\mathrm{Ra}$ relationship, which is expected to take the form of a scaling law

$$
\mathrm{Nu} \sim \mathrm{Ra}^{\alpha}
$$

in the high Rayleigh number limit of fully developed convective turbulence. It is generally assumed that the high Ra scaling law will be independent of the aspect ratio, and independent of the Prandtl number for finite values of Pr. Great interest centers on the asymptotic $($ as $\mathrm{Ra} \rightarrow \infty)$ value of the scaling exponent $\alpha$.

For many applications there is a relatively uncontroversial model of the phenomena, the so-called Boussinesq equations. This model consists of the heat advection-diffusion equation for the local temperature coupled to the incompressible Navier-Stokes equations via a buoyancy force proportional to the local temperature. There have been many theoretical predictions-as well

${ }^{a}$ Electronic mail: const@math.uchicago.edu 
as more than a few a posteriori explanations-for the numerical value of the scaling exponent $\alpha$ based in part on this model. ${ }^{4}$ And while a number of laboratory experiments over the last two decades ${ }^{5-9}$ have produced data yielding clear scaling over many orders of magnitude variation in $\mathrm{Ra}$, experiments have not yet produced unambiguous measurements of $\alpha$. (Directly observed values of $\alpha$ have varied between roughly $1 / 4$ and $1 / 3$.)

One of the early high-Rayleigh number theories ${ }^{10}$ predicted, for finite $\mathrm{Pr}$, an "ultimate" regime as $\mathrm{Ra} \rightarrow \infty$ with $\mathrm{Nu} \sim(\operatorname{Pr} \mathrm{Ra})^{1 / 2}$ (modulo logarithmic modifications). This scaling is distinguished in that the physical heat flux is then independent of the material transport coefficients, ${ }^{1}$ and additionally in that this Rayleigh number dependence is in accord with the most general rigorous upper bounds on the heat transport derived from the Boussinesq equations ${ }^{11}$ with at most mild statistical assumptions. ${ }^{12}$ In particular the best high Ra rigorous bounds to date are of the form $\mathrm{Nu} \leqslant c \mathrm{Ra}^{1 / 2}$ uniform in the Prandtl number for $0<\operatorname{Pr} \leqslant \infty$. Several recent experimental investigations have suggested some indication of the eventual realization of this $\alpha=1 / 2$ limit, ${ }^{6,7}$ but others have concluded that this regime may not be achieved. ${ }^{8,9}$

In this paper we focus on a specific version of the problem modeled by the infinite Prandtl number limit of the Boussinesq equations. Then the inertial terms in the momentum equations are neglected and the velocity vector field is linearly slaved to the temperature field. The infinite Prandtl number limit is the standard model for mantle convection studies ${ }^{2}$ in terrestrial geophysics where $\operatorname{Pr} \approx 10^{24}$, and it is often taken as a reasonable description of high Prandtl number convection at moderate Rayleigh numbers. The Reynolds number is always small for infinite Prandtl number, and the expectation for the high Rayleigh number scaling of the Nusselt number is modified accordingly. The scaling $\mathrm{Nu} \sim \mathrm{Ra}^{1 / 3}$ was predicted on the basis of marginally stable boundary layer arguments, ${ }^{13,14}$ and this value of $\alpha$ is distinguished in that it yields a finite heat flux into a semi-infinite layer. This $1 / 3$ scaling is also predicted as an upper limit of the infinite Prandtl number limit of the Boussinesq equations on the basis of an approximate treatment of an upper bound analysis utilizing mild statistical hypothesis. ${ }^{15}$ More recently, the suggestive high Rayleigh number bound $\mathrm{Nu} \leqslant c \mathrm{Ra}^{1 / 3}(\log \mathrm{Ra})^{2 / 3}$ was proven directly from the equations of motion. ${ }^{16}$

The effect of rotation on convective heat transport is an important issue in astrophysical and geophysical applications, and it has also been the subject of recent laboratory studies ${ }^{17}$ Rotation is modeled by the addition of a Coriolis force to the momentum balance in the Boussinesq model, and introduces another nondimensional variable into the system, the Taylor number Ta, which is proportional to the square of the rotation rate. Rotation modifies the transition from conduction to convection, ${ }^{18,19}$ and generally rotation is observed to suppress convective heat transport in accord with the Taylor-Proudman theorem. The mathematical analysis of the effect of rotation in terms of its effect on rigorous bounds for convective heat transport is only partially successful, however, because to a great extent the existing bounding techniques utilize energy balances. The Coriolis force does no work, so it drops out of the analysis. Indeed, the $\mathrm{Nu} \leqslant c \mathrm{Ra}^{1 / 2}$ bound for arbitrary Prandtl number convection in Refs. 11 and 12 are uniform Ta. To date there are no rigorous estimates of the suppression of convection by rotation for arbitrary Prandtl number fluids.

There has been considerably more success for the analysis of the effect of rotation on infinite Prandtl number convection. When the Coriolis force is introduced directly into the linear slaving of the velocity field to the temperature field, it remains effective when the full momentum equation - and not just the energy balance-is utilized as a constraint in the analysis. A bound on $\mathrm{Nu}$ with the proper qualitative dependence on rotation was recently established, ${ }^{20} \mathrm{Nu}$ $\leqslant c_{1} \mathrm{Ra}^{2} / \sqrt{\mathrm{Ta}}$ for no-slip boundaries. The Rayleigh number dependence of this estimate is overly pessimistic for moderate rotations; in Ref. 21 it is shown that for moderate rotations [Ta $\left.\leqslant c \mathrm{Ra}^{1 / 3}(\log \mathrm{Ra})^{5 / 3}\right]$ an estimate of the form $\mathrm{Nu} \leqslant c \mathrm{Ra}^{1 / 3}(\log \mathrm{Ra})^{2 / 3}$ is valid.

The new results in this paper are to derive another rigorous upper bound for the Nusselt number in the infinite Prandtl number model which is effective for a range of Rayleigh and Taylor numbers. We will prove that

$$
\mathrm{Nu} \leqslant c \mathrm{Ra}^{2 / 5}
$$


where the prefactor $c$ is an absolute constant uniform in Ta. This is a qualitative improvement over the only other known uniform bound $\sim \mathrm{Ra}^{1 / 2}$, and depending on the specific values of $\mathrm{Ra}$ and $\mathrm{Ta}$ it can be a quantitative improvement of the estimates in Refs. 20 and 21. We will establish (1.2) two ways, one in the absence of rotation with the prefactor $c=0.2545 \cdots$, and another in the presence of rotation $^{22}$ with a slightly larger prefactor $c=0.6635 \cdots$.

The rest of this paper is organized as follows. In the next section we present a full description of the Boussinesq model of fluid convection along with the precise definitions of the dependent and independent variables, some basic identities, and a little preliminary analysis. Section III contains the upper bound computation, all the relevant estimates, and two proofs of the 2/5 bound, without and with rotation.

\section{FORMULATION OF THE PROBLEM}

We begin with the Boussinesq model of fluid convection in a rotating reference frame. A layer of fluid is confined between horizontal rigid planes separated by vertical distance $h$. The bottom

plate at $z=0$ is held at constant temperature $T_{\text {bottom }}$, and the top one at $z=h$ is held at temperature $T_{\text {top }}$; both plates are no-slip as regards the fluid motion. The $z$ axis is the vertical direction, the direction in which gravity acts and the direction of the axis of rotation. The unit vectors in the $x$, $y$, and $z$ directions are, respectively, $\mathbf{i}, \mathbf{j}, \mathbf{k}$, and the velocity field is $\mathbf{u}(\mathbf{x}, t)=\mathbf{i} u+\mathbf{j} v+\mathbf{k} w$. The temperature field is $T(\mathbf{x}, t)$. Neglecting compressibility everywhere except in the buoyancy force, and scaling the density to one, the velocity field, the pressure field $p(\mathbf{x}, t)$, and temperature field are governed by the Boussinesq equations

$$
\begin{gathered}
\frac{\partial \mathbf{u}}{\partial t}+\mathbf{u} \cdot \nabla \mathbf{u}+\nabla p+2 \Omega \mathbf{k} \times \mathbf{u}=v \Delta \mathbf{u}+g \alpha \mathbf{k} T, \\
\nabla \cdot \mathbf{u}=0 \\
\frac{\partial T}{\partial t}+\mathbf{u} \cdot \nabla T=\kappa \Delta T .
\end{gathered}
$$

In the above, $\Omega$ is the rotation rate, $v$ is the kinematic viscosity, $g$ is the acceleration of gravity, $\alpha$ is the thermal expansion coefficient, and $\kappa$ is the thermal diffusion coefficient. Incompressibility together with the no-slip boundary conditions lead to the supplementary boundary condition

$$
\frac{\partial w}{\partial z}=0 \quad \text { at } z=0, h .
$$

In this work we restrict attention to periodic boundary conditions on all dependent variables in the horizontal directions with periods $L_{x}$ and $L_{y}$.

The standard nondimensional formulation of the problem is realized by measuring lengths in units of the layer depth $h$, time in units of the thermal diffusion time $h^{2} / \kappa$, velocity in terms of $\kappa / h$, and temperature on a scale where $T_{\text {top }}=0$ and $T_{\text {bottom }}=1$. The equations of motion are then transformed to

$$
\begin{aligned}
& \frac{1}{\operatorname{Pr}}\left(\frac{\partial \mathbf{u}}{\partial t}+\mathbf{u} \cdot \nabla \mathbf{u}\right)+\sqrt{\mathrm{Ta}} \mathbf{k} \times \mathbf{u}+\nabla p=\Delta \mathbf{u}+\operatorname{Ra} \mathbf{k} T, \\
& \nabla \cdot \mathbf{u}=0, \\
& \frac{\partial T}{\partial t}+\mathbf{u} \cdot \nabla T=\Delta T,
\end{aligned}
$$

with boundary conditions 


$$
\left.\mathbf{u}\right|_{z=0}=0=\left.\mathbf{u}\right|_{z=1},\left.\quad T\right|_{z=0}=1,\left.\quad T\right|_{z=1}=0
$$

All the parameters of the system are thus absorbed into four pure numbers. The natural control parameter is the Rayleigh number

$$
\mathrm{Ra}=\frac{g \alpha\left(T_{\text {bottom }}-T_{\text {top }}\right) h^{3}}{v \kappa},
$$

a ratio of the overall buoyancy force to the damping coefficients. The rotation is measured by the Taylor number

$$
\mathrm{Ta}=\left[\frac{2 \Omega h^{2}}{v}\right]^{2}
$$

which is sometimes expressed in terms of the Ekman number $\mathrm{Ek}=\mathrm{Ta}^{-1 / 2}$. The Prandtl number

$$
\operatorname{Pr}=\frac{v}{\kappa}
$$

is a material parameter. In the remainder of this paper we shall be concerned with the infinite Prandtl number model where the inertial terms in the momentum equations (2.5) are dropped so that the velocity field is linearly slaved to the temperature field

$$
\sqrt{\mathrm{Ta}} \mathbf{k} \times \mathbf{u}+\nabla p=\Delta \mathbf{u}+\operatorname{Ra} \mathbf{k} T \text {. }
$$

The fourth pure number characterizing the model is the aspect ratio of the system, which we define to be the nondimensional area on the layer

$$
\mathcal{A}=\frac{L_{x} L_{y}}{h^{2}} .
$$

The infinite Prandtl number model leads to direct linear relationships among the temperature, the vertical velocity $w$, and the vertical vorticity

$$
\zeta=\frac{\partial v}{\partial x}-\frac{\partial u}{\partial y}
$$

Indeed, eliminating the pressure from (2.12) it is straightforward to see that

$$
\Delta^{2} w-\sqrt{\operatorname{Ta}} \frac{\partial \zeta}{\partial z}=-\operatorname{Ra} \Delta_{H} T
$$

where $\Delta_{H}$ denotes the horizontal Laplacian $\partial_{x}^{2}+\partial_{y}^{2}$, and

$$
-\Delta \zeta-\sqrt{\mathrm{Ta}} \frac{\partial w}{\partial z}=0
$$

In view of the incompressibility and no-slip conditions on $\mathbf{u}$, the boundary conditions accompanying (2.15) and (2.16) are

$$
\left.w\right|_{z=0}=0=\left.w\right|_{z=1},\left.\quad \frac{\partial w}{\partial z}\right|_{z=0}=0=\left.\frac{\partial w}{\partial z}\right|_{z=1}
$$

and 


$$
\left.\zeta\right|_{z=0}=0=\left.\zeta\right|_{z=1} .
$$

\section{A. Heat transport}

The total heat transport is the space-time average of the vertical component of the heat current $\mathbf{k} \cdot \mathbf{J}$, where $\mathbf{J}$ is proportional to $\mathbf{u} T-\nabla T$. The standard nondimensional measure of the convective heat transport is the enhancement of the heat flux due to fluid motion, the Nusselt number $\mathrm{Nu}$. The Nusselt number is defined as the ratio of the total vertical heat flux to the conductive heat flux $\kappa\left(T_{\text {bottom }}-T_{\text {top }}\right) / h$.

The convection rate is a time averaged bulk property in the turbulent case, so it is helpful in defining it to introduce the notation

$$
\int_{V} d V=\int_{0}^{L_{x} / h} d x \int_{0}^{L_{y} / h} d y \int_{0}^{1} d z,
$$

(where $x, y$, and $z$ are the nondimensional coordinates) for the volume integration

$$
\|f\|=\left(\int_{V} d V|f(x, y, z)|^{2}\right)^{1 / 2},
$$

for the $L_{2}$ norm on the domain, and

$$
\langle f\rangle=\lim _{t \rightarrow \infty} \frac{1}{t} \int_{0}^{t} f\left(t^{\prime}\right) d t^{\prime},
$$

for the long time average of $f$. In the event the limit above does not exist (or is not unambiguously unique), we may interpret the definition in terms of the limit supremum as regards the upper bounds to be derived here.

Straightforward manipulation of the equations of motion yield a variety of expressions for the Nusselt number in terms of solutions to (2.7), (2.15), and (2.16)

$$
\begin{aligned}
\mathrm{Nu} & =1+\frac{1}{\mathcal{A}}\left\langle\int_{V} d V w T\right\rangle \\
& =\frac{1}{\mathcal{A}}\left\langle\|\nabla T\|^{2}\right\rangle \\
& =\frac{1}{\operatorname{Ra}} \frac{1}{\mathcal{A}}\left\langle\|\nabla \mathbf{u}\|^{2}\right\rangle .
\end{aligned}
$$

The goal of the analysis is to produce a priori bounds for the function $\mathrm{Nu}(\mathrm{Ra}, \mathrm{Ta}, \mathcal{A})$. We will derive bounds that are uniform in the rotation rate and the aspect ratio, so for convenience we will refer to the Nusselt number as simply $\mathrm{Nu}(\mathrm{Ra})$.

\section{B. A useful decomposition}

A device that we shall use throughout is the decomposition of the temperature field into a steady "background profile" and a time-dependent fluctuation field

$$
T(\mathbf{x}, t)=\tau(z)+\theta(\mathbf{x}, t) .
$$

The background profile $\tau(z)$ is, for the moment, arbitrary except that it satisfies the boundary conditions on $T(\mathbf{x}, t)$. That is 


$$
\tau(0)=1 \quad \text { and } \quad \tau(1)=0
$$

Thus the fluctuation $\theta$ satisfies homogeneous boundary conditions

$$
\left.\theta\right|_{z=0}=0=\left.\theta\right|_{z=1},
$$

together with periodicity in the horizontal. A particular background profile will be chosen later for convenience in the analysis.

We introduce the decomposition (2.25) into (2.7) to obtain

$$
\frac{\partial \theta}{\partial t}+\mathbf{u} \cdot \nabla \theta=\Delta \theta-\tau^{\prime} w+\tau^{\prime \prime}
$$

where $\tau^{\prime}(z)$ and $\tau^{\prime \prime}(z)$ are the first and second derivatives of the $\tau(z)$. The evolution of the $L_{2}$ norm (squared) of the fluctuation field is obtained by dotting $\theta$ into Eq. (2.28) and integrating over the volume. Then upon performing some integrations by parts and invoking the boundary conditions we obtain

$$
\frac{d}{d t} \frac{1}{2}\|\theta\|^{2}=-\|\nabla \theta\|^{2}-\int_{V} d V \tau^{\prime}(z) w \theta-\int_{V} d V \tau^{\prime}(z) \frac{\partial \theta}{\partial z}
$$

Now consider the $L_{2}$ norm (squared) of the decomposition of the gradient of the temperature field

$$
\|\nabla T\|^{2}=\mathcal{A} \int_{0}^{1} d z \tau^{\prime}(z)^{2}+2 \int_{V} d V \tau^{\prime}(z) \frac{\partial \theta}{\partial z}+\|\nabla \theta\|^{2} .
$$

Adding $2 \times(2.29)$ to $(2.30)$, taking the long time average and recalling (2.23), we find the fundamental (for what follows) relation for the heat transport:

$$
\mathrm{Nu}=\int_{0}^{1} d z \tau^{\prime}(z)^{2}-\frac{1}{\mathcal{A}}\left\langle\int_{V} d V\left(|\nabla \theta|^{2}+2 \tau^{\prime}(z) w \theta\right)\right\rangle .
$$

\section{UPPER BOUNDS}

The derivation of upper bounds on the convective heat transport is based on the basic decomposition in (2.31). From this starting point we follow two distinct paths to producing effective rigorous estimates for $\mathrm{Nu}(\mathrm{Ra})$. One approach is to choose the background profile $\tau(z)$ to assure non-negativity of the quadratic form

$$
Q\{\theta\}=\int_{V} d V\left(|\nabla \theta|^{2}+2 \tau^{\prime}(z) w \theta\right),
$$

defined for functions $\theta(x, y, z)$ satisfying the fluctuation's boundary conditions with $w(x, y, z)$ given in terms of $\theta$ according to (2.15)—noting that $\Delta_{H} T=\Delta_{H} \theta$ - and (2.16). Then the Nusselt number is bounded explicitly by $\int_{0}^{1} d z \tau^{\prime}(z)^{2}$. The other approach is to derive an a priori upper bound on $\left|\left\langle\int_{V} d V \tau^{\prime}(z) w \theta\right\rangle\right|$ in terms of Ra and the functional form of $\tau$, followed by an appropriate adjustment of $\tau$ to balance this estimate with $\int_{0}^{1} d z \tau^{\prime}(z)^{2}$. We will see that while the first approach can be carried out to derive a bound $\sim \mathrm{Ra}^{2 / 5}$ in the absence of rotation, i.e., for $\mathrm{Ta}=0$, the second approach produces a similarly scaling bound uniform in $\mathrm{Ta}$ for all $-\infty<\mathrm{Ta}<\infty$, albeit with a slightly larger prefactor.

In both approaches the background profile is chosen so that the support of $\tau^{\prime}(z)$ is concentrated near the boundaries where $w$ and $\theta$ are forced to vanish due to the boundary conditions. In particular we take 


$$
\tau(z)=\left\{\begin{array}{l}
1-\frac{z}{2 \delta} \quad \text { for } 0 \leqslant z \leqslant \delta \\
\frac{1}{2} \quad \text { for } \delta \leqslant z \leqslant 1-\delta \\
\frac{1-z}{2 \delta} \quad \text { for } 1-\delta \leqslant z \leqslant 1
\end{array},\right.
$$

where the adjustable parameter $\delta\left(0 \leqslant \delta \leqslant \frac{1}{2}\right)$ is referred to as the "boundary layer" thickness of the profile. Then $\tau^{\prime}(z)$ vanishes in the bulk, and is the constant $-1 / 2 \delta$ within distance $\delta$ of the isothermal boundaries. With this choice of background profile, both approaches rely on detailed local estimates for $\theta$ and $w$ in the boundary layers near $z=0$ and $z=1$.

\section{A. The 2/5 bound without rotation}

First we treat the case of no rotation, i.e., $\mathrm{Ta}=0$. Then the vertical velocity $w$ satisfies

$$
\Delta^{2} w=-\operatorname{Ra} \Delta_{H} \theta,
$$

together with the boundary conditions in (2.17). The procedure is now to show that we may choose the background profile's boundary layer thickness $\delta$ small enough (depending on Ra) to ensure that the quadratic form $Q$ in (3.1) is non-negative. Then the bound will be $\mathrm{Nu} \leqslant 1 / 2 \delta$.

We go over to the Fourier series representation to derive sufficient conditions for the nonnegativity of $Q$. Decomposing $Q$ mode by mode in the translation invariant horizontal directions, we observe that it will be non-negative when for each horizontal wave number $\mathbf{k}$

$$
Q_{\mathbf{k}}\left\{\theta_{\mathbf{k}}\right\}=\int_{0}^{1}\left[\left|D \theta_{\mathbf{k}}\right|^{2}+k^{2}\left|\theta_{\mathbf{k}}\right|^{2}+\tau^{\prime}\left(w_{\mathbf{k}}^{*} \theta_{\mathbf{k}}+w_{\mathbf{k}} \theta_{\mathbf{k}}^{*}\right)\right] d z,
$$

is non-negative. In the above, $\theta_{\mathbf{k}}(z)$ is a complex valued function satisfying $\theta_{-\mathbf{k}}(z)=\theta_{\mathbf{k}}(z)^{*}$ and homogeneous Dirichlet boundary conditions

$$
\theta_{\mathbf{k}}(0)=0=\theta_{\mathbf{k}}(1),
$$

the $z$ derivative is denoted by $D$, and the complex valued function $w_{\mathbf{k}}(z)$ is the linear functional of $\theta_{\mathbf{k}}$ defined by

$$
\left(-D^{2}+k^{2}\right)^{2} w_{\mathbf{k}}=\operatorname{Ra} k^{2} \theta_{\mathbf{k}},
$$

with both homogeneous Dirichlet and Neumann boundary conditions:

$$
w_{\mathbf{k}}(0)=0=w_{\mathbf{k}}(1) \quad \text { and } \quad D w_{\mathbf{k}}(0)=0=D w_{\mathbf{k}}(1) .
$$

Note that $w_{\mathbf{k}}$ also satisfies $w_{-\mathbf{k}}(z)=w_{\mathbf{k}}(z)^{*}$. In this subsection we will also use $\|\cdot\|$ to denote the $L_{2}$ norm and $\|\cdot\|_{\infty}$ to denote the $L_{\infty}$ norm $[0,1]$, i.e.,

$$
\|f\|=\sqrt{\int_{0}^{1}|f(z)|^{2} d z}
$$

and

$$
\|f\|_{\infty}=\sup _{0 \leqslant z \leqslant 1}|f(z)| .
$$

Consider first the temperature fluctuation component $\theta_{\mathbf{k}}(z)$. We estimate the growth of $\theta_{\mathbf{k}}(z)$ in the boundary layer according to 


$$
\left|\theta_{\mathbf{k}}(z)\right|=\left|\int_{0}^{z} D \theta_{\mathbf{k}}\left(z^{\prime}\right) d z^{\prime}\right| \leqslant \sqrt{z} \sqrt{\int_{0}^{1 / 2}\left|D \theta_{\mathbf{k}}\left(z^{\prime}\right)\right|^{2} d z^{\prime}}
$$

for $0 \leqslant z \leqslant \frac{1}{2}$. Of course a similar estimate holds for the growth away from the boundary at $z$ $=1$.

We may obtain control of higher derivatives of $w_{\mathbf{k}}(z)$ in terms of the $L_{2}$ norm of $\theta_{\mathbf{k}}$ which will result in the growth of $w_{\mathbf{k}}(z)$ away from the boundaries being bounded by a higher power of the distance to the wall. Squaring (3.6) and integrating from 0 to 1 , and integrating by parts where the boundary conditions permit, we have

$$
\begin{aligned}
\mathrm{Ra}^{2} k^{4}\left\|\theta_{\mathbf{k}}\right\|^{2}= & \left\|D^{4} w_{\mathbf{k}}\right\|^{2}-2 k^{2} \int_{0}^{1}\left[D^{4} w_{\mathbf{k}}^{*} D^{2} w_{\mathbf{k}}+D^{4} w_{\mathbf{k}} D^{2} w_{\mathbf{k}}^{*}\right] d z \\
& +6 k^{4}\left\|D^{2} w_{\mathbf{k}}\right\|^{2}+4 k^{6}\left\|D w_{\mathbf{k}}\right\|^{2}+k^{8}\left\|w_{\mathbf{k}}\right\|^{2} .
\end{aligned}
$$

The indefinite term above may be estimated by its neighboring terms. For any $a>0$

$$
\left|2 k^{2} \int_{0}^{1}\left[D^{4} w_{\mathbf{k}}^{*} D^{2} w_{\mathbf{k}}+D^{4} w_{\mathbf{k}} D^{2} w_{\mathbf{k}}^{*}\right] d z\right| \leqslant a\left\|D^{4} w_{\mathbf{k}}\right\|^{2}+\frac{4 k^{4}}{a}\left\|D^{2} w_{\mathbf{k}}\right\|^{2} .
$$

Choosing $a=\frac{1}{2}(\sqrt{41}-5) \approx 0.7016$, then, we see that

$$
\operatorname{Ra}^{2} k^{4}\left\|\theta_{\mathbf{k}}\right\|^{2} \geqslant C\left[\left\|D^{4} w_{\mathbf{k}}\right\|^{2}+k^{4}\left\|D^{2} w_{\mathbf{k}}\right\|^{2}\right]
$$

where $C=\frac{1}{2}(7-\sqrt{41}) \approx 0.2984$. This will be enough to give us $L_{\infty}$ control of $D^{2} w_{\mathbf{k}}$ in light of the following:

Lemma: Let $f(z)$ be a smooth (say, $D^{3} f$ is continuous) real valued function satisfying both homogeneous Dirichlet and Neumann boundary conditions on $[0,1]$. Then

$$
\left\|D^{2} f\right\|_{\infty} \leqslant \sqrt{2\left\|D^{4} f\right\|\left\|D^{2} f\right\|} .
$$

We note that this lemma is not true unless both sets of boundary conditions are satisfied. Counterexamples are the functions $f(z)=2 z^{3}-3 z^{2}+z$ and $f(z)=2 z^{3}-3 z^{2}+1$ which satisfy, respectively, homogeneous Dirichlet and homogeneous Neumann boundary conditions on $[0,1]$. Each of these functions has $\left\|D^{2} f\right\|_{\infty}=6$ although $\left\|D^{4} f\right\|=0$.

To prove the lemma, first note that because of the homogeneous Dirichlet conditions, $\int_{0}^{1} D f(z) d z=0$, so $D f$ must have a zero inside the interval $(0,1)$. That is, there exists a point $z_{0}$ $\in(0,1)$ so that $D f\left(z_{0}\right)=0$. Then because of the homogeneous Neumann conditions, $\int_{0}^{z_{0}} D^{2} f(z) d z=0$ and $\int_{z_{0}}^{1} D^{2} f(z) d z=0$ so there exist points $z_{1} \in\left(0, z_{0}\right)$ and $z_{2} \in\left(z_{0}, 1\right)$ so that $D^{2} f\left(z_{1}\right)=0=D^{2} f\left(z_{2}\right)$.

So with $0<z_{1}<z_{2}<1$ being distinct zeros on $D^{2} f$, we use the fundamental theorem of calculus to write

$$
\left(D^{2} f(z)\right)^{2}=2 \int_{z_{1}}^{z} d z^{\prime} D^{2} f\left(z^{\prime}\right) D^{3} f\left(z^{\prime}\right)
$$

and, for any point $\tilde{z} \in(0,1)$

$$
D^{3} f\left(z^{\prime}\right)=D^{3} f(\widetilde{z})+\int_{\tilde{z}}^{z^{\prime}} d z^{\prime \prime} D^{4} f\left(z^{\prime \prime}\right)
$$

Inserting (3.16) into (3.15) we have 


$$
\left(D^{2} f(z)\right)^{2}=2 D^{3} f(\widetilde{z}) \int_{z_{1}}^{z} d z^{\prime} D^{2} f\left(z^{\prime}\right)+2 \int_{z_{1}}^{z} d z^{\prime} D^{2} f\left(z^{\prime}\right) \int_{\tilde{z}}^{z^{\prime}} d z^{\prime \prime} D^{4} f\left(z^{\prime \prime}\right) .
$$

Integrating (3.17) with respect to $\widetilde{z}$ from $z_{1}$ to $z_{2}$ and noting that the first term on the right hand side vanishes, we deduce

$$
\left(z_{2}-z_{1}\right)\left(D^{2} f(z)\right)^{2}=2 \int_{z_{1}}^{z_{2}} d \widetilde{z} \int_{z_{1}}^{z} d z^{\prime} D^{2} f\left(z^{\prime}\right) \int_{\tilde{z}}^{z^{\prime}} d z^{\prime \prime} D^{4} f\left(z^{\prime \prime}\right)
$$

The Schwarz inequality (applied twice) then implies

$$
\left(z_{2}-z_{1}\right)\left(D^{2} f(z)\right)^{2} \leqslant 2\left(z_{2}-z_{1}\right)\left\|D^{2} f\right\|\left\|D^{4} f\right\|,
$$

which proves the lemma.

Returning attention to $w_{\mathbf{k}}(z)=u(z)+i v(z)$ with $u$ and $v$ real (and each satisfying the boundary conditions in the lemma) and recalling (3.13), we have

$$
\begin{aligned}
\left|D^{2} w_{\mathbf{k}}(z)\right|^{2} & =\left(D^{2} u(z)\right)^{2}+\left(D^{2} v(z)\right)^{2} \\
& \leqslant 2\left\|D^{2} u\right\|\left\|D^{4} u\right\|+2\left\|D^{2} v\right\|\left\|D^{4} v\right\| \\
& \leqslant k^{2}\left\|D^{2} u\right\|^{2}+\frac{1}{k^{2}}\left\|D^{4} u\right\|^{2}+k^{2}\left\|D^{2} v\right\|^{2}+\frac{1}{k^{2}}\left\|D^{4} v\right\|^{2} \\
& =k^{2}\left\|D^{2} w_{\mathbf{k}}\right\|^{2}+\frac{1}{k^{2}}\left\|D^{4} w_{\mathbf{k}}\right\|^{2} \\
& \leqslant \frac{\mathrm{Ra}^{2}}{C} k^{2}\left\|\theta_{\mathbf{k}}\right\|^{2} .
\end{aligned}
$$

Hence the growth of $w_{\mathbf{k}}$ from the boundary at $z=0$ is limited by

$$
\begin{aligned}
\left|w_{\mathbf{k}}(z)\right| & =\left|\int_{0}^{z} d z^{\prime} \int_{0}^{z^{\prime}} d z^{\prime \prime} D^{2} w_{\mathbf{k}}\left(z^{\prime \prime}\right)\right| \\
& \leqslant \frac{1}{2} z^{2}\left\|D^{2} w_{\mathbf{k}}\right\|_{\infty} \\
& \leqslant \frac{1}{2} z^{2} \frac{\mathrm{Ra}}{\sqrt{C}} k\left\|\theta_{\mathbf{k}}\right\| .
\end{aligned}
$$

An analogous pointwise bound holds near the boundary at $z=1$.

The magnitude of the indefinite term in $Q_{\mathbf{k}}$ is then bounded in terms of the positive definite terms: 


$$
\begin{aligned}
\int_{0}^{1} \tau^{\prime}\left(w_{\mathbf{k}}^{*} \theta_{\mathbf{k}}+w_{\mathbf{k}} \theta_{\mathbf{k}}^{*}\right) d z \leqslant & \frac{1}{\delta} \int_{0}^{\delta}\left|w_{\mathbf{k}}(z)\left\|\theta_{\mathbf{k}}(z)\left|d z+\frac{1}{\delta} \int_{1-\delta}^{1}\right| w_{\mathbf{k}}(z)\right\| \theta_{\mathbf{k}}(z)\right| d z \\
\leqslant & \frac{1}{\delta} \int_{0}^{\delta} d z \frac{1}{2} z^{2}\left(\frac{\mathrm{Ra}}{\sqrt{C}} k\left\|\theta_{\mathbf{k}}\right\|\right) \sqrt{z} \sqrt{\int_{0}^{1 / 2}\left|D \theta_{\mathbf{k}}\left(z^{\prime}\right)\right|^{2} d z^{\prime}} \\
& +\frac{1}{\delta} \int_{1-\delta}^{1} d z \frac{1}{2}(1-z)^{2}\left(\frac{\mathrm{Ra}}{\sqrt{C}} k\left\|\theta_{\mathbf{k}}\right\|\right)\left|\theta_{\mathbf{k}}(z)\right| \sqrt{1-z} \sqrt{\int_{1 / 2}^{1}\left|D \theta_{\mathbf{k}}\left(z^{\prime}\right)\right|^{2} d z^{\prime}} \\
\leqslant & \frac{1}{7} \delta^{5 / 2} \frac{\operatorname{Ra}}{\sqrt{C}} k\left\|\theta_{\mathbf{k}}\right\|\left(\sqrt{\int_{0}^{1 / 2}\left|D \theta_{\mathbf{k}}\left(z^{\prime}\right)\right|^{2} d z^{\prime}}+\sqrt{\int_{1 / 2}^{1}\left|D \theta_{\mathbf{k}}\left(z^{\prime}\right)\right|^{2} d z^{\prime}}\right) \\
\leqslant & \frac{1}{7} \delta^{5 / 2} \frac{\operatorname{Ra}}{\sqrt{C}} \frac{1}{\sqrt{2}}\left(k^{2}\left\|\theta_{\mathbf{k}}\right\|^{2}+\left\|D \theta_{\mathbf{k}}\right\|^{2}\right) .
\end{aligned}
$$

Hence $Q_{\mathbf{k}}$ is non-negative for all $\mathbf{k}$ when we choose $\delta$ so that

$$
1=\frac{1}{7 \sqrt{2 C}} \delta^{5 / 2} \mathrm{Ra}
$$

The heat transport is then bounded according to

$$
\mathrm{Nu} \leqslant \frac{1}{2 \delta}=\frac{1}{2(98 C)^{1 / 5}} \mathrm{Ra}^{2 / 5}=0.2545 \cdots \mathrm{Ra}^{2 / 5} .
$$

This proof does not go through when $\mathrm{Ta} \neq 0$, however, because we cannot establish the highest derivative control in (3.13).

\section{B. The 2/5 bound with rotation}

In the presence of rotation we adopt another strategy to derive the $2 / 5$ scaling bound, albeit with a slightly larger prefactor. When $\mathrm{Ta} \neq 0$ we cannot use the tight $z^{2}$ bound on $w$ near the boundary because we cannot produce an effective estimate for the fourth derivative of $w$ in $L_{2}$. We can, however, establish a $z^{3 / 2}$ growth. Then we do not require that the quadratic form $Q$ be non-negative, but rather we find an a priori estimate for $Q$ and then adjust the background boundary layer thickness $\delta$ to make the best of it.

Consider first the temperature field. Because $T(\mathbf{x}, t)$ solves the advection-diffusion equation (2.7), it satisfies a maximum principle. That is, if the initial data $T(\mathbf{x}, 0)$ is bounded pointwise between the values at the boundaries, i.e., if $0 \leqslant T(\mathbf{x}, 0) \leqslant 1$, then the solution subsequently sustains those limits: $0 \leqslant T(\mathbf{x}, t) \leqslant 1$. The background profile in (3.2) is also bounded pointwise in magnitude between 0 and 1 , so the fluctuation $\theta$ also obeys the same limits. Hence

$$
\|T\|_{\infty}=\|\tau\|_{\infty}=1 \quad \text { and } \quad\|\theta\|_{\infty} \leqslant 1 .
$$

We now establish growth limits on $w$ near the boundaries as follows: The vertical components of velocity and vorticity are slaved to the temperature fluctuations by

$$
\Delta^{2} w-\sqrt{\mathrm{Ta}} \frac{\partial \zeta}{\partial z}=-\operatorname{Ra} \Delta_{H} \theta
$$

and 


$$
-\Delta \zeta-\sqrt{\mathrm{Ta}} \frac{\partial w}{\partial z}=0
$$

Multiplying (3.26) by $w$ and (3.27) by $\zeta$, integrating over the full domain, and integrating by parts with the help of the boundary conditions (2.17) and (2.18)

$$
\left\|\frac{\partial^{2} w}{\partial z^{2}}\right\|^{2}+2\left\|\nabla_{H} \frac{\partial w}{\partial z}\right\|^{2}+\left\|\Delta_{H} w\right\|^{2}+\|\nabla \zeta\|^{2}=\operatorname{Ra} \int_{V} d V \theta\left(-\Delta_{H} w\right) \leqslant \frac{\mathrm{Ra}^{2}}{4}\|\theta\|^{2}+\left\|\Delta_{H} w\right\|^{2},
$$

where, not unexpectedly, $\nabla_{H}$ denotes the horizontal gradient $\mathbf{i}(\partial / \partial x)+\mathbf{j}(\partial / \partial y)$. Then thanks to (3.25), the second vertical derivative of the vertical velocity is bounded according to

$$
\left\|\frac{\partial^{2} w}{\partial z^{2}}\right\|^{2} \leqslant \frac{\mathrm{Ra}^{2}}{4}\|\theta\|^{2} \leqslant \frac{\mathrm{Ra}^{2}}{4} \mathcal{A}
$$

Then in view of $w^{\prime}$ s boundary conditions, for $0 \leqslant z \leqslant \frac{1}{2}$

$$
|w(x, y, z)|=\left|\int_{0}^{z} d z^{\prime} \int_{0}^{z^{\prime}} d z^{\prime \prime} \frac{\partial^{2} w}{\partial z^{2}}\right| \leqslant \frac{2}{3} z^{3 / 2} \sqrt{\int_{0}^{1 / 2} d z^{\prime \prime}\left(\frac{\partial^{2} w}{\partial z^{2}}\right)^{2}}
$$

A similar estimate holds near the top boundary at $z=1$.

Combining (3.25), (3.29), and (3.30), the indefinite (last) term on the right-hand side of

$$
\mathrm{Nu}=\int_{0}^{1} d z \tau^{\prime}(z)^{2}-\frac{1}{\mathcal{A}}\left\langle\|\nabla \theta\|^{2}\right\rangle-\frac{2}{\mathcal{A}}\left\langle\int_{V} d V \tau^{\prime}(z) w \theta\right\rangle
$$

is seen to satisfy

$$
\begin{aligned}
\int_{V} d V \tau^{\prime}(z) w \theta \leqslant & \frac{1}{2 \delta} \int_{0}^{L_{x} / h} d x \int_{0}^{L_{y} / h} d y \int_{0}^{\delta} d z|w(x, y, z)|\|\theta\|_{\infty} \\
& +\frac{1}{2 \delta} \int_{0}^{L_{x} / h} d x \int_{0}^{L_{y} / h} d y \int_{1-\delta}^{1} d z|w(x, y, z)|\|\theta\|_{\infty} \\
\leqslant & \frac{1}{2 \delta} \frac{2}{3} \frac{2}{5} \delta^{5 / 2} \sqrt{2 \mathcal{A}}\left\|\frac{\partial^{2} w}{\partial z^{2}}\right\| \\
\leqslant & \frac{\sqrt{2}}{15} \delta^{3 / 2} \operatorname{Ra} \mathcal{A}
\end{aligned}
$$

Hence

$$
\mathrm{Nu} \leqslant \frac{1}{2 \delta}+\frac{2 \sqrt{2}}{15} \delta^{3 / 2} \mathrm{Ra}
$$

Now we minimize the right-hand side above with respect to $\delta$ by choosing

$$
\delta=\frac{5^{2 / 5}}{2^{3 / 5}} \mathrm{Ra}^{-2 / 5}
$$

to conclude that 


$$
\mathrm{Nu} \leqslant \frac{5^{3 / 5}}{3 \times 2^{2 / 5}} \mathrm{Ra}^{2 / 5}=0.6635 \cdots \mathrm{Ra}^{2 / 5}
$$

This bound is independent of the rotation, i.e., uniform in Ta for $0 \leqslant \mathrm{Ta}<\infty$, and valid so long as the initial temperature data lie between the temperatures at the boundaries.

\section{ACKNOWLEDGMENTS}

This research was supported in part by awards from the U.S. National Science Foundation to the Universities of Michigan and Chicago. We thank the Isaac Newton Institute for Mathematical Sciences, Cambridge, for their hospitality during the time part of this work was completed. We are grateful to Jesse Otero, Edriss Titi, and Emmanuel Villermaux for helpful suggestions and remarks.

\footnotetext{
${ }^{1}$ E. A. Spiegel, Annu. Rev. Astron. Astrophys. 9, 323 (1971).

${ }^{2}$ See, for example: P. E. Van Keken, Earth Planet. Sci. Lett. 148, 1 (1997).

${ }^{3}$ J. Pedlosky, Geophysical Fluid Dynamics (Springer, Berlin, 1979).

${ }^{4}$ E. Siggia, Annu. Rev. Fluid Mech. 26, 137 (1997).

${ }^{5}$ F. Heslot, B. Castaing, and A. Libchaber, Phys. Rev. A 36, 5870 (1987).

${ }^{6}$ S. Cioni, S. Ciliberto, and J. Sommeria, J. Fluid Mech. 335, 111 (1997).

${ }^{7}$ X. Chavanne, F. Chilla, B. Castaing, B. Hebral, B. Chabaud, and J. Chaussy, Phys. Rev. Lett. 79, 3648 (1997).

${ }^{8}$ J. Glazier, T. Segawa, A. Naert, and M. Sano, Nature (London) 398, 307 (1999).

${ }^{9}$ J. Niemela, L. Skrbek, K. R. Sreenivasan, and R. J. Donnelly, Nature 404, 837-840 (2000).

${ }^{10}$ R. Kraichnan, “Turbulent Thermal Convection at Arbitrary Prandtl Number,' Phys. Fluids 5, 1374 (1962).

${ }^{11}$ C. Doering and P. Constantin, Phys. Rev. E 53, 5957 (1996).

${ }^{12}$ L. N. Howard, J. Fluid Mech. 17, 405 (1963); for a review see: L. N. Howard, Annu. Rev. Fluid Mech. 4, 473 (1972).

${ }^{13}$ W. V. R. Malkus, Proc. R. Soc. London, Ser. A 225, 196 (1954).

${ }^{14}$ L. N. Howard, in Applied Mechanics, Proc. 11th Cong. Applied Mech., edited by H. Görtler (Springer-Verlag, Berlin, 1966), pp. 1109-1115.

${ }^{15}$ S.-K. Chan, Stud. Appl. Math. 50, 13 (1971).

${ }^{16}$ P. Constantin and C. R. Doering, J. Stat. Phys. 94, 159 (1999).

${ }^{17}$ T. H. Rossby, J. Fluid Mech. 36, 309 (1969); Y. Liu and R. Ecke, Phys. Rev. Lett. 79, 2257 (1997).

${ }^{18}$ S. Chandrasekhar, Hydrodynamic and Hydromagnetic Stability (Oxford University Press, Oxford, 1961); P. Drazin and W. Reid, Hydrodynamic Stability (Cambridge University Press, Cambridge, 1981).

${ }^{19}$ R. A. Worthing, Phys. Lett. A 237, 381 (1998).

${ }^{20}$ P. Constantin, C. Hallstrom, and V. Putkaradze, Physica D 125, 275 (1999).

${ }^{21}$ P. Constantin, C. Hallstrom, and V. Putkaradze, J. Math. Phys. 42, 773 (2001).

${ }^{22}$ P. Constantin, Contemp. Math. 238, 77 (1999).
} 